\title{
Design of Reliable Communication Protocol Based on Emergency Satellite Communications Ground Station
}

\author{
Leilei KANG 1st \\ School of Information Science and Engineering \\ Hebei University of Science and Technology \\ Shijiazhuang, China \\ lei-736@163.com \\ Xiaozhe ZHENG 3rd \\ School of Information Science and Engineering \\ Hebei University of Science and Technology \\ Shijiazhuang, China \\ Zhengxiaozhe12@163.com
}

\begin{abstract}
According to the characteristics of satellite emergency communication, combined with the UDP protocol and the TCP protocol of the advantages and disadvantages, the article puts forward an improved expanded with reissue of double reliable D - RCUDP protocol. This paper mainly introduces the improved D-RCUDP protocol application background, the hierarchical structure, the core mechanism, application platform and experimental results.
\end{abstract}

Keywords-UDP; Satellite Emergency Communication; Reliability

\section{INTRODUCTION}

When a sudden disaster occurs, conventional terrestrial communications equipment or systems will have been seriously damaged, so here is the use of the satellite emergency communication system (Figure 1). Satellite user ground stations use the UDP protocol, but it can not guarantee the reliable transmission of data. Therefore, this paper mainly design according to the satellite emergency communication characteristics of based on UDP the application level of the reliable data transfer protocol, and used for the rapid configuration user satellite ground station, so as to ensure the critical information of timely transmission.

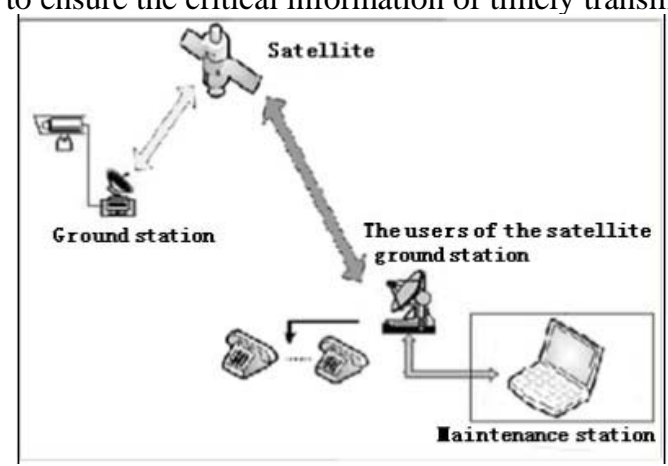

Figure 1. Satellite emergency communication system.

\author{
Xiangyang XU 2nd \\ School of Information Science and Engineering \\ Hebei University of Science and Technology \\ Shijiazhuang, China \\ xuxiangyang@hebust.edu.cn \\ Bingyang CHENG 4th \\ School of Information Science and Engineering \\ Hebei University of Science and Technology \\ Shijiazhuang, China \\ 13703313380@163.com
}

\section{BASED ON THE UDP PROTOCOL OF EXPANDED WITH REISSUE OF DOUBLE RELIABLE D - RCUDP PROTOCOL OF THE DESIGN}

\section{A. Basic principle}

The article mainly studies the reliable data transfer protocol for satellite emergency communication, which has a requirement of speed and reliability, but in the data transmission of process, the contradiction between the speed and reliability can not be eliminated. In other words, highspeed simple transport protocol often has poor reliability; on the contrary the high reliability protocol of transport speed is usually not ideal. Although in the communication process, adding some reliable mechanisms like serial mechanism, CRC check, identify mechanisms, dual-thread mechanism, and keep-alive mechanism, stopping to wait mechanism, retransmission mechanism, discarded errors and duplication package, to ensure its reliability [1]. But stopping to wait mechanism and retransmission mechanism will reduce the transmission speed, and simply discarding the received packet will bring a heavy workload to the retransmission. Sliding window mechanism in TCP protocol is very good, but a bit complicated. So it was suggested that the idea of replacement pointer, although the mere reissue operation efficiency is high, but if the receiving end is not enough processing capacity, or the reissued queue is too long, it will take up a lot of the memory, and affect other operations. So combined with this mechanism, to improve stopping to wait mechanism and retransmission mechanism, and add the queue of the out-of-order queue. This will not only ensure the reliability, but also improve efficiency, and reduce the sending time-consuming, and not simply discard the packet received.

\section{B. Protocol hierarchy}

D-RCUDP protocol based on message is a reliable data transfer protocol, with UDP protocol as the underlying 
support protocol [2], the D-RCUDP protocol hierarchy as shown in Figure 2.

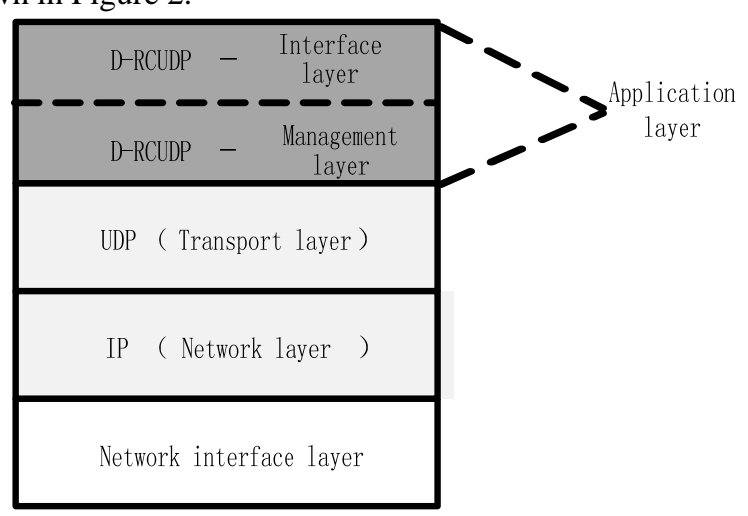

Figure 2. D-RCUDP hierarchical structure diagram.

D-RCUDP - the interface layer is responsible for sending and receiving, and passes the data to management layer analysis. D-RCUDP - the management layer main for the protocol content of analysis and processing, for example: recognition analysis, reliable data processing analysis and maintaining links keep-alive message processing.

\section{Protocol basic}

The D-RCUDP protocol uses the means of communication should be in conformity with the relevant provisions. This protocol adapted to communicate both IP address on the same subnet, is not restricted to specific IP address. The underlying transport protocol using UDP protocol, maintenance monitor as a server $(\mathrm{CON})$, satellite ground station as a user client (PUS).

\section{Internal core mechanism}

1) with patulous and reissue of double reliable mechanism.

In the maintenance station initial configuration of the satellite users ground stations, in order to make the fast into the work of the state, will be normal to send, reproduction and expanding reissue for a kind of new combination operation, that is to say in normal send uninterrupted cases to join repeat and expanded reissue combining the mechanism, and based on not simple give up the data packets of principle, add out-of-order mechanism. Because the stop wait mechanism and retransmission mechanism can make the transmission speed is reduced, and the received packet simply discarded, to repeat great workload. Also because of simple replacement operation efficiency is high, but if the receiver processing capacity is not enough, or the replacement queue is too long will take up a lot of memory, but affect other operations. Therefore, through the analysis of two mechanism the malpractice, stopped waiting mechanism and retransmission mechanism is improved, and joined the out-of-order queue, so can have assure reliable basis, improve efficiency, reduce the transmission time consuming, not simply abandon the received packet. Specific design as shown in figure 3 below:

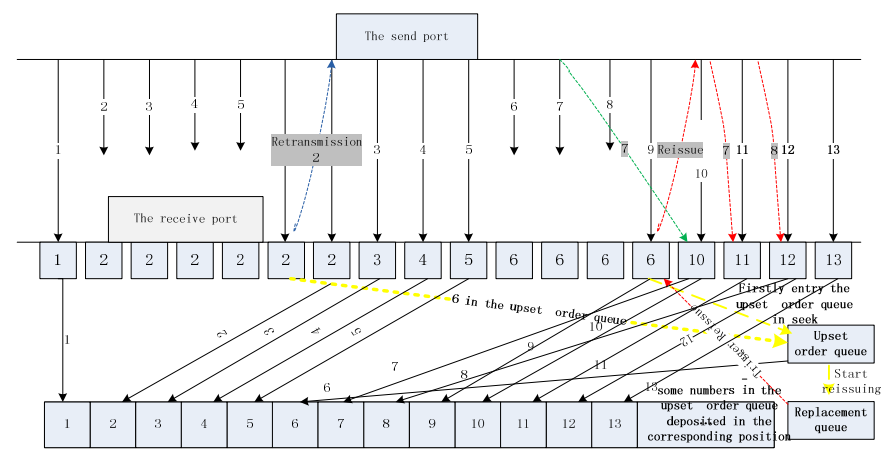

Figure 3. With the development of a reliable mechanism for double.

When received data packet number and required number difference is greater than $\mathrm{K}$ (Take $\mathrm{K}=3$ ), trigger retransmission mechanism, as shown in figure 4,1 packets of normal and deposited into the corresponding position, after should receive 2 pack, but received 6 packet, and the required number of difference for 4 . This shows that $2,3,4$, 5 received packets are not normal, then start the retransmission mechanism, the pointer to rescheduled for 2 packets, and to send the correct number, meanwhile will received receive 6 out-of-order packets into local out-oforder data buffer queue, and set out-of-order sequence data counter. When the receiving end more than threshold clear out-of-order queue, in order to avoid take up a lot of memory which affect transmission efficiency. The sending end received in a retransmission request according to the correct number repositioning transmits the location, from the current package began retransmitting data.

Continue above the transmission process, from the 6 package began, when packet's number with the desired data number difference is greater than 0 , but less than or equal to $\mathrm{K}$, trigger expanded with reissue mechanism. As shown in Figure 3, the receiving end receives the pass over the data packets through the analytically derived number is 9 , but need the number is 6 , the difference between the two is 3 . Then do not need to send again, only in the normal transmission on the basis of turn on the expanded with reissue mechanism, and the number of offset storage, save to correspond to the number 9 position. At the same time in out-of-order queue search need $6,7,8$ pack, if the search to the need pack is stored to a specified location, without triggering the extended reissue pointer. If there is no need to bag, then sends expanded reissue request to the sending end, the sending end after receipt of a request does not change the original position is transmitted, but through expanded reissue mechanism proceed expand reissue package fast positioning and replacement treatment, namely open two threads. After searching out-of-order queue exist 6 pack, the 6 pack save to the corresponding position, but no search to 7 , 8 bag. Now that enabled the two threads, a thread in a sending normal package No.10 while another thread send expanded reissue request required 7, 8 pack.

When parsing a number with the desired number difference is less than 0 , the message is expanded reissue 
package or a duplicate packet, the receiving end on the documents search, if the corresponding number place vacant, is to expand the reissue package, whereas for a duplicate packet, discard. As shown in Figure 3, expanded reissue request needs 7,8 pack. In 7 is received packet check the corresponding position have data, indicates that the packet has been received, the packet to a duplicate packet, can be discarded directly. In 8 is received packet check the corresponding position without the data storage, the bag to expand the reissue package, should be stored to correspond to the location.

When analyzing the package number with the package number equal, the right to receive the message, carries on the corresponding storage operation.

$\mathrm{K}$ is an adjustable threshold, can according to the actual situation for lowering and rising. If required interface will reduce the $K$ value, in order to reduce reissue increasing retransmission. If want fast transmission, can improve the $\mathrm{K}$ value, increase the reissue and reduce retransmission.

When the satellite ground station started to work after, if the maintenance station needs to carry on the parameter modification and query, because this does not need huge amounts of data, but must ensure that the changes of the parameters of accuracy, so to switch to the modified stop waiting mechanism.

Link in UP state, then an improved stop waiting for mechanism. This mechanism ensures that the receiving end receives the accurate news. In sending before not received the response message (retransmission times is not more than 5), unable to do the next message to send. Received the same serial of information processing only the first, the others only confirmation response. Communication of both sides to maintain his serial number $C$ field, the range of $0 \sim 127$ circulation, receives the message and verify correct, the number $\mathrm{C}$ field of the seventh position to set 1 , composition the response frame to send to each other, and record the current received to the opposite end $\mathrm{C}$ field value, if once again received the same serial number message only response without treatment [3].

\section{2) Link switching detection mechanism.}

Keep-Alive message used to link the on-off detection. Keep alive messages and data messaging by port: the sender (CON) uses port 6911, the receiving end (PUS) using 6611 port. Initialize the transceiver serial numbers are 0 , state are DOWN, KEEP_ALIVE cycle for 3 seconds, 3 successive KEEP_ALIVE send after no response, that link DOWN, and clear the queue, suspension Keep-Alive timing, wait for the new connection, otherwise send new keep-alive message.

\section{D-RCUDP PROTOCOL APPLICATION PLATFORM}

\section{A. Introduction for maintenance station}

Portable satellite ground station maintenance is an essential part of the equipment, through the D - RCUDP communication protocol to complete the planning of the use of ground stations, configuration management, day-to-day maintenance and condition monitoring, with the functions to save, retrieve, and bill the call record [4]. As shown in Figure 4 below:

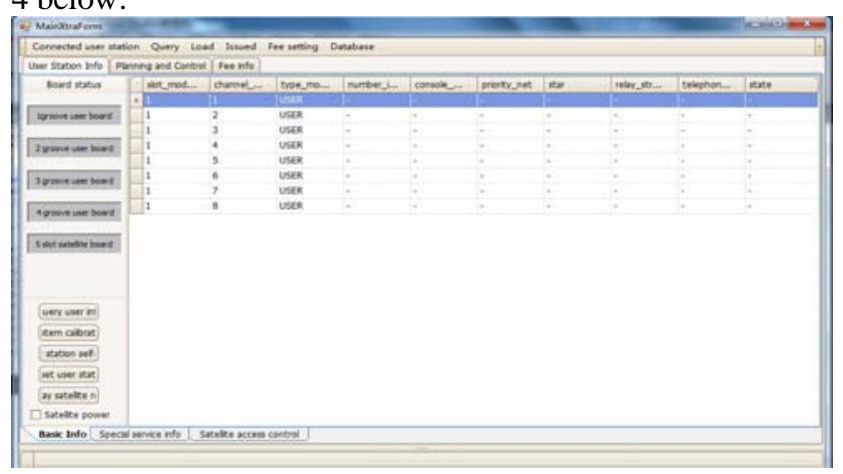

Figure 4. Satellite ground station maintenance console user interface.

\section{B. The basic functions of the maintaining station}

1) Configuration management function: Completing the configuration of the user attributes, conferences and satellite module, with functions of configuration data separate file generation and load.

2) Condition monitoring: Monitoring the state of the user station and completing its self-test and self-test result processing functions.

3) Billing function: Completing the call records acquisition, storage, retrieval and billing functions.

4) Configuration data export functions: Completing to get the data from the user station configuration and configuration data alone generates configuration files.

5) Multi-user station management: Maintenance station can manage a plurality of user stations (up to 32), to select the corresponding data according to the ID of the user station to manage only one subscriber station at the same time.

\section{THE TEST RESULTS AND ANALYSIS}

Using the software Wireshark to get the transmission of data and analysis, is able to draw the D-RCUDP agreement to ensure correct data transmission. In accordance with the data collected in the experiment, generate the curve shown in Figure 5. The figure shows the transmission efficiency curve of the TCP, UDT (UDP-based Data Transfer Protocol) and D-RCUDP three protocols in the same network environment [5]. The following conclusions can be drawn from Figure 5: First, D-RCUDP protocol to transmit large amounts of data in a short period of time has obvious advantages. The speed of the D-RCUDP agreement advantage is more and more obvious with the increase of the transmission of data. Second in the public network environment, D-RCUDP Agreement has not received a poor environment impact visible the D-RCUDP agreement is not the quality of the network environment. 


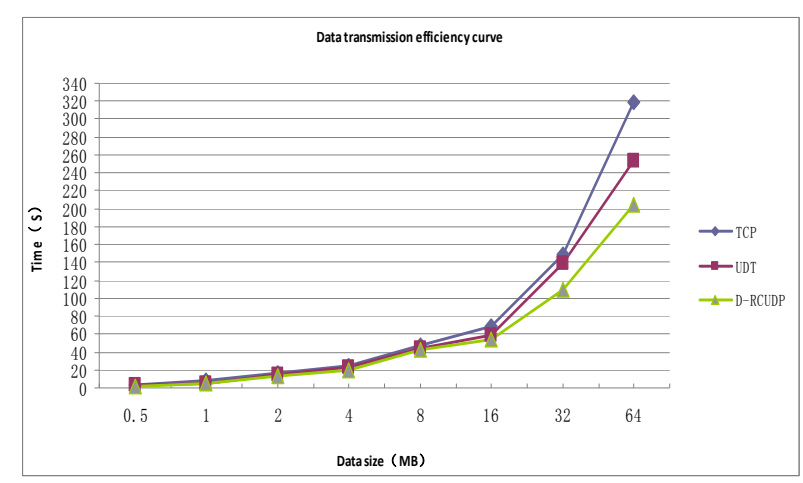

Figure 5. Data transmission efficiency curve.

At the same time in the laboratory simulation tests, the frame rate of the D-RCUDP agreement loss is zero when it is under in 16, 64, 128 byte frame. In the actual emergency communication process, the frame loss rate is less than $2 \%$, related to the actual link quality and transmission interference, but it is able to resolve this problem through dual reliably expand reissue.

\section{CONCLUSION}

With emergency satellite communication technology matures, the study of large-capacity high-speed interactive communication necessary to data reception has also been a new development. In emergency communication process In above the udp protocol to join the double reliable communication protocol D - RCUDP, makes the reliability and efficiency have been give attention to two or morethings, so that the reliability is assured, without reducing the transmission efficiency also. With further research, the outof-order queue threshold settings problem further highlighted. If too short, it will not achieve a big role. If too long, it is a burden for memory. Therefore, research and optimization this problem to ensure satellite communications and ground data receiving and distribution system more perfect and practical is the next task.

\section{ACKNOWLEDGMENT}

During this period, I received much help and support from my classmates and my teacher. Especially my teacher, Xiangyang $\mathrm{XU}$, give me much guidance from researching protocol to designing and perfecting of the system. In here, I am grateful to them.

\section{REFERENCES}

[1] W. Xiaoli, ”Satellite radio environment reliable transmission protocol design and implementation", Computer Systems \& Applications, vol.1, PP.63-66, April 2010.

[2] J.Postel, User Datagram Protocol. RFC768, 1980.

[3] W. Yufeng, "Use UDP to realize tata reliable transmission Mechanism”, The Ministry 54 Institute, vol.1, PP.29-32, January 1996.

[4] L. Mingbo, C\# Senior Programming, 4th ed., Beijing: Tsinghua university press, 2006, PP.153-334.

[5] S. Hyuck Leea, S. Seokb, C. Gu Kangb and C. Kangb, ”The two markers system for TCP and UDP fows in a differentiated services", Network Computer Communications, vol. 2, PP.338-350, July 2003. 